\title{
The Coulomb Pump: a Novel Parts Feeding Method using a Horizontally-Vibrating Surface *
}

\author{
Dan Reznik \\ John Canny \\ EECS Dept., UC-Berkeley, CA 94720-1776 USA
}

\begin{abstract}
Part feeders, which singulate and orient parts prior to packing and insertion, are critical components of an assembly line. Existing feeders utilize off-plane vibrations of a rigid structure to convey parts along a track. Repeated part hopping/landing phases are concerns if parts are delicate and/or high positioning accuracy is required. Here we consider a simpler feeder design in which parts are in permanent contact with a horizontally-vibrating flat plate. Each vibration is a "pump-like" motion along a single degree of freedom: the plate spends more time moving forward than backward. Parts are propelled forward since dynamic friction is fixed and independent of the relative velocity at the interface. In designing plate vibration profles we consider issues of waveform simplicity, bandwidth, and feed rate performance. Both bang-bang and sinusoidal control waveforms are analyzed. Expressions are derived for equilibrium feed rates for both waveforms; dynamic simulation is used to verify the analysis. A prototype of the proposed feeder has been implemented with cheap mechanical parts. A simple experiment with the device is presented.
\end{abstract}

\section{Introduction}

Part feeders, which singulate and orient parts prior to packing and insertion, are critical components of an assembly line and one of the biggest obstacles to flexible assembly. Vibratory bowl feeders use $3 d$ vibrations of a rigid bowl to feed industrial parts along a helical track [1]. In each vibration cycle, a part undergoes sticking, hopping, landing, and sliding motion modes. In such applications one is not concerned with part positioning accuracy nor with premature part wear due to repeated collisions with the feeder.

In the spirit of minimalism [2], we consider a simpler vibrations-based parts feeder design based on a horizontally vibrating flat plate, as shown in Figure 1. A linear motor (e.g., a voice coil) accelerates the plate along a single degree of freedom. Parts lay flush with the plate; force is transferred to the part via dynamic friction (assuming the plate vibrates fast enough). This reduces part wear (assuming sliding friction is better than rigid collisions), and improves part motion controllability.

Each plate vibration is an asymmetric, pump-like closed motion along a single degree of freedom: the plate spends

* Support provided in part by NSF grant CDA-9726389: "Challenges in CISE: Planning and Control for Massively Parallel Manipulation"

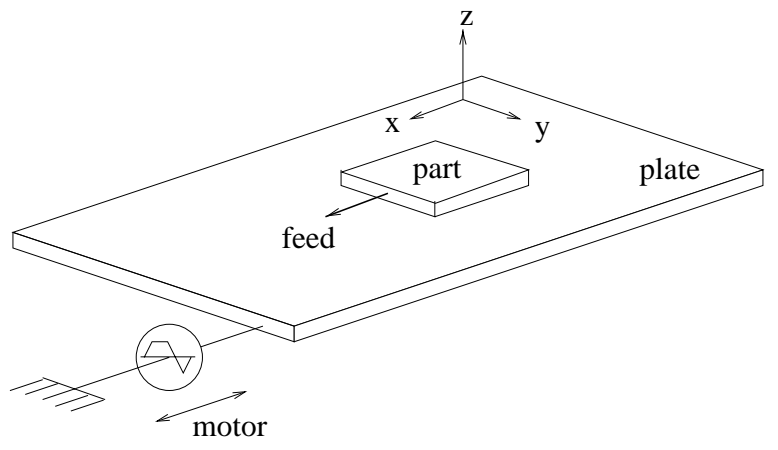

Figure 1: Our proposed feeder design: a flat plate, actuated by a linear motor, vibrates along a single degree of freedom. Parts lay flush with the plate. The plate's pump-like motion results in positive net dynamic frictional force applied to the part per cycle, causing the part to feed forward.

more time moving forward than backward. Since dynamic frictional forces are independent of the relative velocity at the part-plate interface, this type of motion results in positive net force applied to the part per cycle, resulting in forward feeding.

In designing plate vibration profiles, i.e., the waveform input to the motor, we consider issues of wave simplicity (ease of synthesis) and low bandwidth (anti-resonance). Two "canonical" waveforms types are analyzed: bang-bang and sinusoidal. In the former, the motor is driven at either full or zero throttle; in the latter, input to the motor consists a low frequency sine plus its first harmonic. Analytic expressions for both feeding forces and feed rates are derived. A feed rate performance measure is defined which allows for some quantitative comparison of the two methods. Results for the sinusoidal method are informally verified with dynamic simulation.

A prototype of the feeder has been built with inexpensive parts. A simple part feeding experiment is presented.

\subsection{Related Work}

Zesch et al. have developed a micro-positioning device which steps over a stationary flat plate by contracting/extending its body [3]. Similar to inch-worm motion, this device exploits both stiction and sliding frictional modes. In a recent work [4], we show that asymmetry in the compressiondecompression phases of part-actuator impact is the primary 
cause for forward part feeding for a type of MEMs motion array [5]. Other examples of array-based feeders which exploit asymmetry include [6, 7]. Hayward [8] has investigated the interaction of simple rigid parts placed on a horizontally/vertically vibrating plate: by modulating the motion control waveforms, parts automatically orient/position themselves at energy minima. Böhringer et al. [9] have proposed to use the vibration nodes to automatically gather particles at specific locations.

The ideas presented here relate to our ongoing work on 2d part manipulation with a horizontally-vibrating surface, described in the companion paper [10]. In that work, we show that a closed motion of the plate (involving its 3 degrees of planar freedom) can be computed which generates arbitrary forces (averaged over one oscillation cycle) at a finite number of points, allowing for the parallel motion control of one or more parts.

The remainder of this paper is organized as follows: In Section 2 we present the part feeder's mechanical model and the principle of pump-like motion, or "time-asymmetry". In Section 3, we consider motion waveform design and compare two possible controls: bang-bang and sinusoidal. In Section 4 we present a simple experiment performed with a prototype of the feeder. Conclusions follow in Section 5.

\section{Part Feeding Principles}

\section{$2.1 \quad$ Feeder model}

The parts feeder we consider is illustrated in Figure 2. A waveform generator $W$ produces a periodic signal which is power-amplified $(A)$ and fed to a linear motor $M$. The motor converts input energy into output force $f_{e x t}$, applied along $x$ to a flat plate $S$. The plate is constrained to move along a single horizontal dimension (e.g., $x$ ), with gravity acting perpendicularly (along $-z$ ). The plate's $x$ compliance (suspension mechanism) is modeled as a damped-spring $(k, b)$. One or more parts $P$ are placed over the plate's surface.

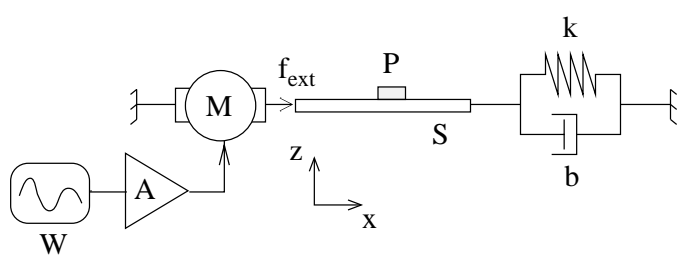

Figure 2: Parts feeder model: a flat plate $S$ is actuated along $x$ by a linear motor $M$; the plate's compliance/suspension is modeled as a damped spring $(k, b) . M$ converts the amplified signal generated by $W$ into output force $f_{e x t}$ applied to $S$ along $x$.

Let $a_{s}(t)=f_{e x t} / m_{s}$ denote the plate's instantaneous acceleration, where $m_{s}$ is the plate's mass (parts' masses are negligible). In what follows we will ignore amplification and motor transfer function issues and assume $a_{s}(t)$ can be specified directly. Let $a_{s}(t)$ be a periodic function with period $T$. The feeder is a mass-spring system with resonance $w_{0}$ occurring at [11]:

$$
w_{0}=\sqrt{\frac{k}{m_{s}}-\left(\frac{b}{4 k m_{s}}\right)^{2}}
$$

Imposing $T \ll 2 \pi / w_{0}$, i.e., $a_{s}(t)$ well above resonance, affords us the following nice properties:

- Plate oscillations are bounded.

- The plate's velocity profile $\nu_{s}(t)$ is the perfect timeintegral of $a_{s}(t)$ and has zero steady-state DC level [11].

- Surface-part relative accelerations are above the threshold of sticking ${ }^{1}$ [12], i.e., $P$ is always sliding on $S$.

The plate's surface is assumed smooth and with uniform coefficient of dynamic friction $\mu$. Let $\nu_{s}(t), \nu_{p}(t)$ denote the plate's and part's instantaneous velocities, respectively. The Coulomb model for dynamic friction [12] states that the instantaneous frictional force acting on $P$ will be of fixed magnitude $\mu \mathrm{mg}$ and act opposite to the relative velocity, i.e.:

$$
f_{\text {fric }}(t)=\mu m g \operatorname{sgn}\left[\nu_{s}(t)-\nu_{p}(t)\right]
$$

\subsection{Time Asymmetry}

In order for a part to feed forward, it must perceive a positive net frictional force as interacts with the plate over one oscillation cycle. The non-linear dependency of frictional force on relative velocity (in fact, the former's independence on the latter) suggests a simple approach to achieve part feeding: choose $a_{s}(t)$ such that $\nu_{s}(t)$ is positive for a longer time than it is negative. We term such a $\nu_{s}(t)$ time-asymmetric. For simplicity's sake, we make the following quasi-static assumption: the maximum change in part's speed in one cycle $\mu g T$ is negligible compared to the plate's peak velocity, $\nu_{s, \max }$. Since the latter is bounded by $a_{\max } T$, where $a_{\max }$ is the peak acceleration in one cycle, this requirement corresponds loosely to imposing $\mu g / a_{\max } \ll 1$, which is facilitated by either one of: a slippery surface, a lightweight plate, and/or a powerful motor.

Let $\nu_{p}$ denote the part's "constant" velocity in one cycle. From Equation 2, we obtain an expression for the average force $f_{1 d}$ applied to the part over one cycle:

$$
\bar{f}_{1 d}=\frac{\mu m g}{T} \int_{0}^{T} \operatorname{sgn}\left[\nu_{s}(t)-\nu_{p}\right] d t
$$

The effect of the sgn function above is to "saturate" $\nu_{s}(t)-\nu_{p}$, i.e., transform it into a square wave. Define $t^{+}$as the duration of the positive portion of $\nu_{s}(t)-\nu_{p}$. The average force will be linked to the asymmetry in the $\operatorname{sgn}\left[\nu_{s}(t)-\nu_{p}\right]$ square wave, i.e., to $t^{+}$'s deviation from $T / 2$. It can be shown that:

$$
\bar{f}_{1 d}=\mu m g\left(\frac{2 t^{+}}{T}-1\right)
$$

If looked at the scale of several cycles, the part's velocity will increase if the force applied to it per cycle is non-zero, i.e., $t^{+}>T / 2$. This process will converge when the part reaches an equilibrium speed $\nu_{e q}$, called the feed rate, such that $\bar{f}_{1 d}=0$. The concepts in this Section are illustrated in Figure 3 .

\footnotetext{
${ }^{1}$ Actually, short periods of sticking, ignored here, will occur near the zero-crossings of relative acceleration
} 


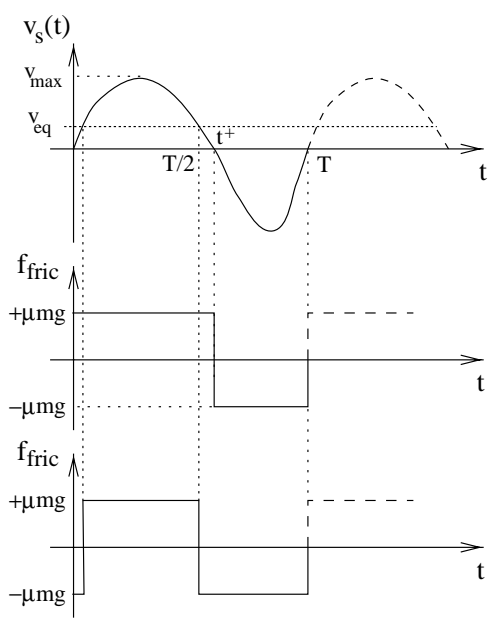

Figure 3: (a) A time-asymmetric velocity profile $\nu_{s}(t)$ : the length of its positive semi-cycle is greater than $T / 2 ; \nu_{s}(t)$ 's peak value is $\nu_{\max }$. If the part's velocity is zero, $t^{+}>T / 2$. (b) Instantaneous frictional forces for a null part velocity: $\nu_{s}(t)-\nu_{p}$ is "saturated" into an asymmetric square wave. (c) The frictional force at equilibrium is a square wave with $50 \%$ duty cycle. The corresponding part velocity $\nu_{e q}$ is shown in (a) as the horizontal line which divides $\nu_{s}(t)$ into positive and negative semi-cycles of equal length $T / 2$.

\section{Designing the Control Waveform}

In designing an $a_{s}(t)$ which achieves a time-asymmetric $\nu_{s}(t)$ we look for the following properties:

- Simplicity: a functionally simple $a_{s}(t)$ is easy to synthesize and parameterize.

- Bandwidth: low harmonic content reduces the chance of feeder resonance. Also, the motor will greatly attenuate $a_{s}(t)$ 's high frequency components.

- Performance: one measure of performance is the ratio of the feed rate $\nu_{e q}$ by $\nu_{s}(t)$ 's peak value. Since this is bounded by $a_{\max } T$, and will typically correspond to $\nu_{s}(t)$ 's negative extremum $\left|\nu_{\min }\right|$, we define the following two performance measures:

$$
\begin{aligned}
& \lambda_{1}=\nu_{e q} /\left(a_{\max } T\right) \\
& \lambda_{2}=\nu_{e q} /\left|\nu_{\min }\right|
\end{aligned}
$$

In what follows we present two $a_{s}(t)$ waveforms. The first focuses on waveform simplicity, while the second attempts to minimize spectral content. We then compare their $\lambda_{1,2}$ performances.

\subsection{Bang-bang acceleration}

A simplifying assumption is to expect that the motor can only deliver three types of forces: full throttle forward, zero force, and full throttle backward. As it turns out, if these three force application modes are repeated in sequence, the plate will move in a time-asymmetric manner. The corresponding $a_{s}(t)$ is the "bang-bang" waveform shown in Figure 4(a), and defined below:

$$
\begin{aligned}
a_{s}(t) & = \begin{cases}a_{\max } & 0 \leq t<t_{1} \\
0 & t_{1} \leq t<t_{2} \\
-a_{\max } & t_{2} \leq t<T\end{cases} \\
t_{1} & =(1-z) T / 2 \\
t_{2} & =(1+z) T / 2
\end{aligned}
$$

Where $z \in[0,1)$ is a parameter governing the zero-force phase duration. Namely, $a_{s}(t) 3$ phases are: (i) positive constant acceleration, (ii) zero-acceleration, and (iii) negative constant acceleration. For simplicity's sake, we let phases (i) and (iii) be of equal duration; phase (ii)'s length is $z T . a_{\max }$ is the (bounded) acceleration desired in phases (i) and (iii). Integration (and removal of the DC term) yields a piecewise linear expression for the velocity profile:

$$
\begin{aligned}
\nu_{s}(t) & = \begin{cases}a_{\max }\left[\frac{T}{4}\left(z^{2}-1\right)+t\right] & 0 \leq t<t_{1} \\
a_{\max } \frac{T}{4}(z-1)^{2} & t_{1} \leq t<t_{2} \\
a_{\max }\left[\frac{T}{4}\left(z^{2}+3\right)-t\right] & t_{2} \leq t<T\end{cases} \\
t_{1}, t_{2} & =\text { as in above. }
\end{aligned}
$$

The above function is illustrated in Figure 4.
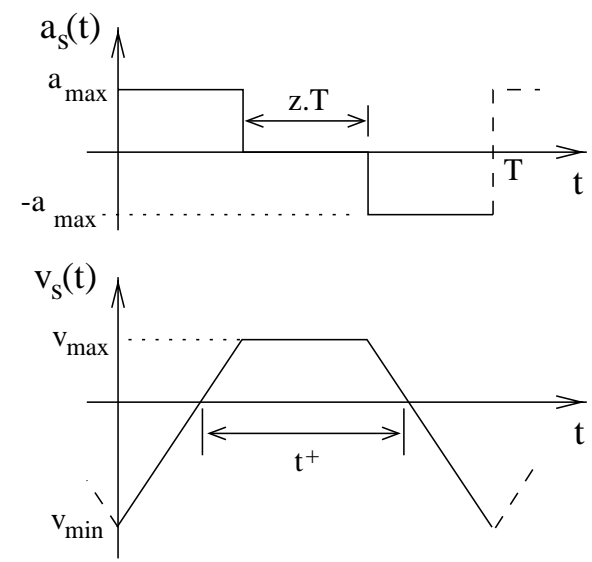

(b)

Figure 4: (a) A "bang-bang" acceleration profile with $z=$ $1 / 3$. (b) The corresponding velocity profile $\nu_{s}(t)$ shown with zero steady state DC value (due to springiness in the suspension). Shown also is the $t^{+}$, the length its positive semicycle.

The following expressions yield $\nu_{s}(t)$ 's maximum and minimum values:

$$
\begin{aligned}
& \nu_{\min }=a_{\max } T \frac{\left(z^{2}-1\right)}{4}<0 \\
& \nu_{\max }=a_{\max } T \frac{(z-1)^{2}}{4}>0
\end{aligned}
$$

For a fixed part velocity $\nu_{p}$, we derive an expression for $t^{+}$ in $\nu_{s}(t)-\nu_{p}$ :

$$
t^{+}=\frac{T}{2}\left(z^{2}+1\right)-\frac{2 \nu_{p}}{a_{\max }}
$$

From Equation 4 obtain the average force applied to the part per cycle: 


$$
\bar{f}_{1 d}=\mu m g\left(z^{2}-\frac{\nu_{p}}{a_{\max } T}\right)
$$

The equilibrium velocity $\nu_{e q}$ is obtained by solving $\bar{f}_{1 d}=0$, above, for $\nu_{p}$ :

$$
\nu_{e q}=a_{\max } T \frac{z^{2}}{4}
$$

The above equation is only valid for $z<1 / 2$. Beyond $1 / 2$, $\nu_{e q}$ is "clamped" by $\nu_{\max }$ (see [13] for details), and decreases to zero as $z$ approaches 1 , as shown in Figure 5.

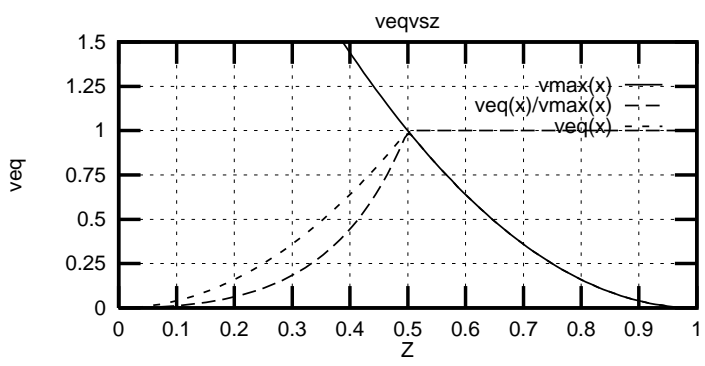

Figure 5: The feed rate $\nu_{e q}$ plotted against the $z$ parameter, for $a_{\max }=16$, and and $T=1$. Shown also the graphs of $\nu_{\max }$ and the ratio $r=\nu_{e q} / \nu_{\max }$. Notice that if $z>1 / 2$, $\nu_{e q}$ tracks $\nu_{\max }$.

A maximum feed rate of $a_{\max } T / 16$ is achieved at $z=1 / 2$ so $\lambda_{1}=.0625$. Plugging $z=1 / 2$ into Equation 8 , obtain $\lambda_{2}=1 / 3$ for this type of control waveform.

\subsubsection{Viscous force model}

Taking the derivative of $\bar{f}_{1 d}$ in Equation 10 with respect to $\nu_{p}$ and substituting in the value of $\nu_{e q}$ from Equation 11, yields an interesting expression for the average frictional force as a function of part speed:

$$
\begin{aligned}
\bar{f}_{1 d} & =K\left(\nu_{e q}-\nu_{p}\right) \\
\text { with } K & =\frac{4 \mu m g}{a_{\max } T}
\end{aligned}
$$

So in the average sense, the part perceives the plate (under bang-bang control) as a viscous fluid flowing forward at the feed rate, i.e., given enough time, the part's speed will approach that of the fluid.

\subsection{Sinusoidal acceleration}

Trivially, a cosine-like $a_{s}(t)$ integrates to a symmetric velocity profile. The lowest bandwidth $a_{s}(t)$ which integrates to a time-asymmetric waveform is a cosine plus a scaled, phase-shifted, double-frequency cosine:

$$
\begin{aligned}
& a_{s}(t)=\cos (t)+2 b \cos (2 t+\phi) \\
& \nu_{s}(t)=\sin (t)+b \sin (2 t+\phi)
\end{aligned}
$$

where $b$ and $\phi$ are arbitrary ${ }^{2}$. Equation 4 links $\bar{f}_{1 d}$ to the roots of $\nu_{s}(t)-\nu_{p}$. It can be shown that Equation 14

\footnotetext{
${ }^{2}$ Interestingly, $a_{s}(t)=\cos (t)+b \cos (3 t-\phi)$ integrates to a velocity profile with a symmetry of the type $\nu_{s}(t)=-\nu_{s}(-\pi+t)$, yielding zero feeding force for any $b, \phi$.
}

will have either 2 or 4 real roots. Let us label these in ascending order in the $[-\pi, \pi)$ interval as $r_{i}, i=1 \ldots 4$. Without loss of generality, assume that $a_{s}\left(r_{1}\right)$ is positive. So the length of $\nu_{s}(t)$ 's positive portion will be simply the sum of the distances between consecutive root pairs, i.e., $t^{+}=\left(r_{2}-r_{1}\right)+\left(r_{4}-r_{3}\right)$. By expressing each root as a function of $b$ and $\phi$ we can derive an expression for $\bar{f}_{1 d}$ using Equation 4. In particular, with $\phi=\pi / 2$, i.e., $\nu_{s}(t)=\sin (t)+b \cos (2 t)$ we derive:

$$
\begin{aligned}
& \bar{f}_{1 d}=-\mu m g \frac{2}{\pi} c_{1}+ \begin{cases}-1+\frac{2}{\pi} c_{2} & b>1 \\
0 & |b|<1 \\
+1-\frac{2}{\pi} c_{2} & b<1\end{cases} \\
& c_{1}=\sin ^{-1}\left(\frac{1-\sqrt{1+8 b^{2}}}{4 b}\right) \\
& c_{2}=\sin ^{-1}\left(\frac{1+\sqrt{1+8 b^{2}}}{4 b}\right)
\end{aligned}
$$

A good approximation for the $|b|<1$ range is $f_{1 d} \cong b / 3$. To derive an expression for $\nu_{e q}$, we need to consider the roots of $\nu_{s}(t)-\nu_{e q}$, in particular we want $t^{+}=T / 2$. Careful analysis yields a surprisingly simple result:

$$
\nu_{e q}=b \sin \phi,|b|<1 / 2
$$

At $|b|>1 / 2$, the number of roots of $\nu_{s}(t)-\nu_{e q}$ jumps from 2 to 4 causing $\nu_{e q}$ to decay, similar to saturation at $z>1 / 2$ in the bang-bang case. In particular, for $\phi=\pi / 2$, we obtain:

$$
\nu_{e q}= \begin{cases}b & |b|<1 / 2 \\ \frac{1}{4 b} & |b| \geq 1 / 2\end{cases}
$$

At $b=1 / 2$ and $\phi=\pi / 2$, i.e., $\nu_{s}(t)=\sin (t)+\cos (2 t) / 2$, the feed rate is maximal ${ }^{3}$. $\bar{f}_{1 d}$ and $\nu_{e q}$ versus $b$, with $\phi=\pi / 2$, is plotted in Figure 6.

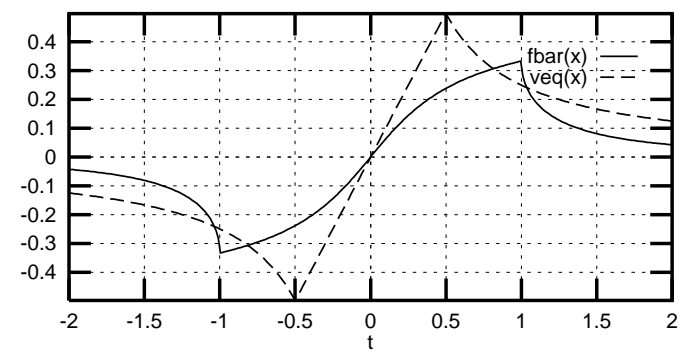

Figure 6: $\bar{f}_{1 d}$ and $\nu_{e q}$ plotted against parameter $b$, for $\nu_{s}(t)=\sin (t)+b \cos (2 t)$. Notice that these quantities are maximal at $b=1$, and $b=1 / 2$, respectively.

Consider the more general expressions for the $\nu_{e q}$-optimal acceleration and velocity profiles:

$$
\begin{aligned}
a_{s, \text { opt }}(t) & =\frac{a_{\max }}{\gamma}[\cos (\omega t)-2 b \sin (2 \omega t)] \\
\nu_{s}(t) & =\frac{a_{\max }}{\gamma}[\sin \omega t+b \cos (2 \omega t)]
\end{aligned}
$$

\footnotetext{
${ }^{3}$ Equation 17 curiously implies that the "cousin" function $\cos (t)+$ $b \sin (2 t)(\phi=-\pi)$ yields zero feed rate, for any choice of $b$.
} 
With $\omega=2 \pi / T$. The constant $\gamma$ is the maximum absolute value of $\cos (t)+2 b \sin (2 t)$. It normalizes $a_{s, \text { opt }}(t)$ so $a_{\max }$ becomes the actual maximum acceleration. Its value is given by:

$$
\begin{array}{r}
\gamma=\frac{\left(3+c_{1}\right) \sqrt{c_{1}+64 b^{2}-1}}{32 \sqrt{2}} \\
c_{1}=\sqrt{1+128 b^{2}}
\end{array}
$$

At $b=1 / 2, \gamma \cong 1.76$. The derived maximum, minimum, and equilibrium levels for this function are as follows:

$$
\begin{aligned}
\nu_{\min } & =-a_{\max } T \frac{3}{4 \pi \gamma} \\
\nu_{\max } & =a_{\max } T \frac{3}{8 \pi \gamma} \\
\nu_{e q} & =a_{\max } T \frac{1}{4 \pi \gamma}
\end{aligned}
$$

Yielding $\lambda_{1}=.0452$ and $\lambda_{2}=1 / 3$. Interestingly, the contraction in harmonic content afforded by the sinusoidal drive results in no penalty for $\lambda_{2}$ and a small reduction in $\lambda_{1}$, relative to the bang-bang method.

\subsubsection{Dynamic simulation}

Dynamic simulation was used to visualize part motion under sinusoidal acceleration (similar results for the bang-bang case can be found in [13]). Part motion is obtained through numerical integration of the instantaneous frictional forces corresponding to a $\nu_{s}(t)=\sin (t)+b \sin (2 t+\phi)$ velocity profile. In Figure 7 , the part speed vs. time is shown for various combinations of $b$ and $\phi$. The thickness of each curve is related to the variability in part speed during each cycle. As apparent, part speed approaches $\nu_{e q}$ as time advances. Figures $8(\mathrm{a}, \mathrm{b})$ illustrate this phenomenon at a much smaller (per-cycle) scale. Namely, part speed becomes, at equilibrium, a triangular waveform of positive (resp. negative) derivative when $\nu_{p}<\nu_{s}(t)$ (resp. $\left.\nu_{p}<\nu_{s}(t)\right)$.

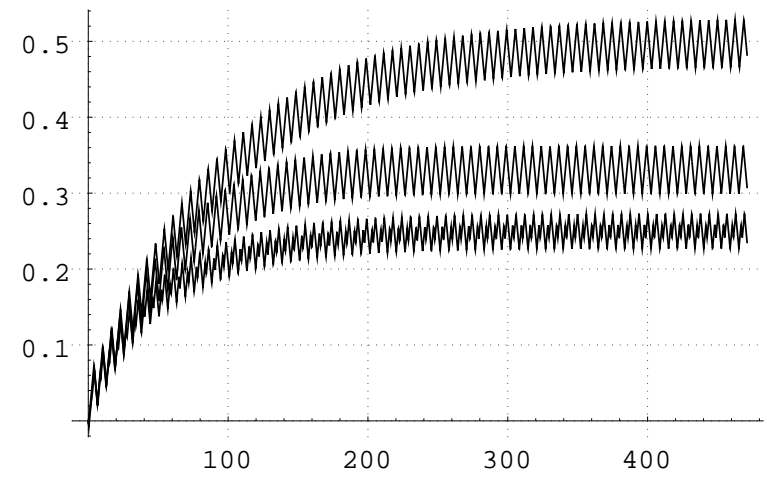

Figure 7: Dynamic simulation of part motion: under $\nu_{s}(t)=$ $\sin (t)+b \sin (2 t+\phi)$, the part's velocity is plotted against time for $(b, \phi)=\left\{\left(\frac{1}{2}, \frac{\pi}{2}\right),\left(1, \frac{\pi}{2}\right),\left(\frac{1}{2}, \frac{\pi}{4}\right)\right\}$, corresponding to the top, middle, and lower curves. Each curve shows the part accelerating from zero velocity to the predicted feed rates $\left(\frac{1}{2}, \frac{1}{3}, \frac{1}{4}\right)$. The curves' "thickness" reveal the change in part speed per cycle.

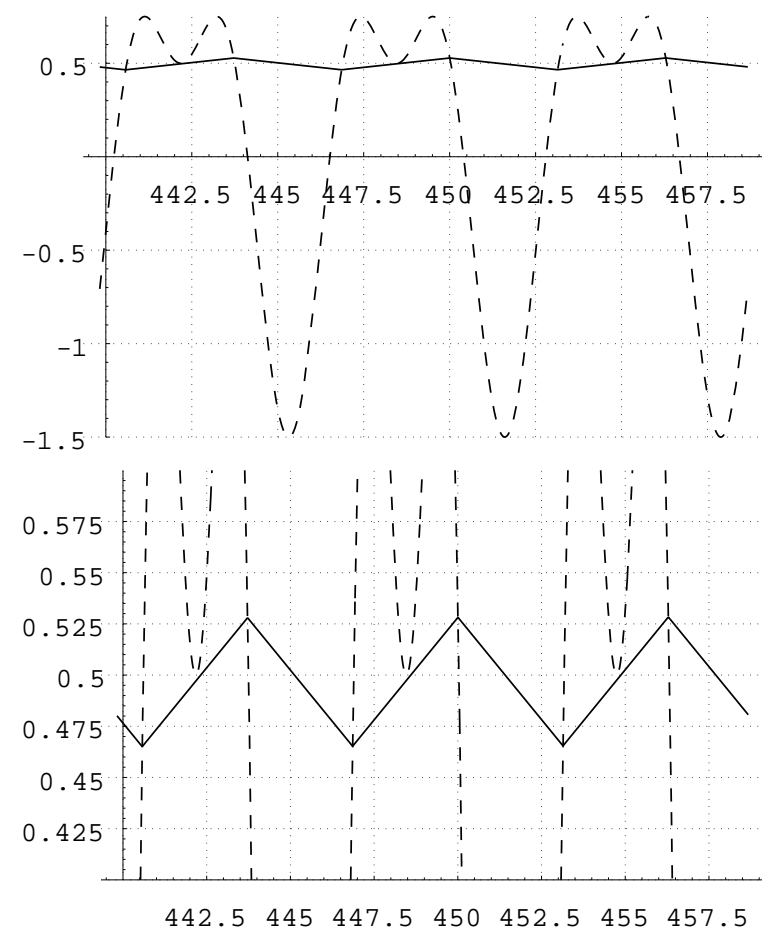

Figure 8: The part's speed (solid line) shown superimposed on the velocity profile (dashed line), for $(b, \phi)=\left(\frac{1}{2}, \frac{\pi}{2}\right)$, at the equilibrium level $\nu_{e q}=\frac{1}{2}$. Top: part speed variability is small compared to $\nu_{\max }$. Bottom: part acceleration/deacceleration visible at a smaller scale.

\section{Hardware Experiments}

A prototype of the parts feeder built out of inexpensive parts is shown in Figure 9. A thin slab of lightweight, smooth, kitchen-top material is used as the plate. Four motors are attached to the plate (one per side) by springy brass shafts, forming a combined suspension/actuation mechanism. Each motor is a voice coil removed from old hard disks. The motors can command horizontal plate vibrations along its 3 dof's - this feature is used in our work on parallel part manipulation [10]. To achieve 1d vibrations, two opposing motors are turned off, while the other two opposing ones are operated in tandem, at 180 degrees phase shift. Figure 10 shows snapshots of a part feeding experiment - the bangbang acceleration profile is used with $z=1 / 3$ and $1 / T=$ $30 \mathrm{~Hz}$. Parts propel forward at about $1 \mathrm{~cm} / \mathrm{s}$.

\section{Conclusion}

With respect to existing hop-based vibratory feeders, our design is mechanically simpler, reduces part wear caused by repeated collisions, and increases part motion controllability. Guidelines based on simplicity, bandwidth, and feed rate performance have been defined in designing a "good" control waveform. The sinusoidal method, despite its minimal bandwidth, results in a small performance loss with respect to the bang-bang control method. A sinusoidal waveform is more likely to be used in practice since it allows us to pre-compensate for both the amplifier's and the motor's frequency response. Quantitative experiments with the feeder's 


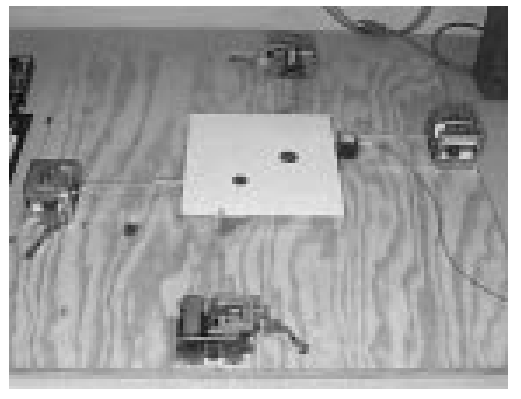

Figure 9: Our feeder's prototype: the plate is a thin square slab of kitchen-top material. Four disk drive motors are attached to the plate through thin brass shafts. Two coins are shown on the plate.

prototype are the next step on this work. In particular, feed rates have to be measured and matched with the analytical results as waveform parameters (e.g., phase and amplitude relationship in sinusoidal case) are varied.

\section{Acknowledgments}

This work was supported by NSF grant CDA-9726389: "Challenges in CISE: Planning and Control for Massively Parallel Manipulation".

\section{References}

[1] G. Boothroyd. Assembly automation and product design. Marcel Dekker, Inc., New York, NY, 1991.

[2] J. Canny and K. Goldberg. RISC for industrial robots: Recent results and open problems. In IEEE International Conference on Robotics and Automation, San Diego, CA, May 1994.

[3] W. Zesch, R. Büchi, and R. Siegwart. Inertial mechanisms for positioning microobjects: two novel mechanisms. In SPIE conference on microrobots and $m i$ cromechanical systems, pages $80-88$, Philadelphia, PA, October 1995.

[4] D. Reznik, S. Brown, and J. Canny. Dynamic simulation as a design tool for a microactuator array. In IEEE International Conference on Robotics and Automation, Albuquerque, NM, April 1997.

[5] K. Böhringer, B. Donald, R. Mihailovich, and N. MacDonald. Sensorless manipulation using massively parallel microfabricated actuator arrays. In IEEE International Conference on Robotics and Automation, San Diego, CA, May 1994.

[6] S. Konishi and H. Fujita. A conveyance system using air flow based on the concept of distributed micro motion systems. Journal of Micro-electromechanical Systems, 3(2):54-58, June 1994 .

[7] J. Suh, S. Glander, R. Darling, C. Storment, and G. Kovacs. Combined organic thermal and electrostatic omnidirectional ciliary microactuator array for object positioning and inspection. In Proc. Solid State Sensor and Actuator Workshop, Hilton Head, NC, June 1996.

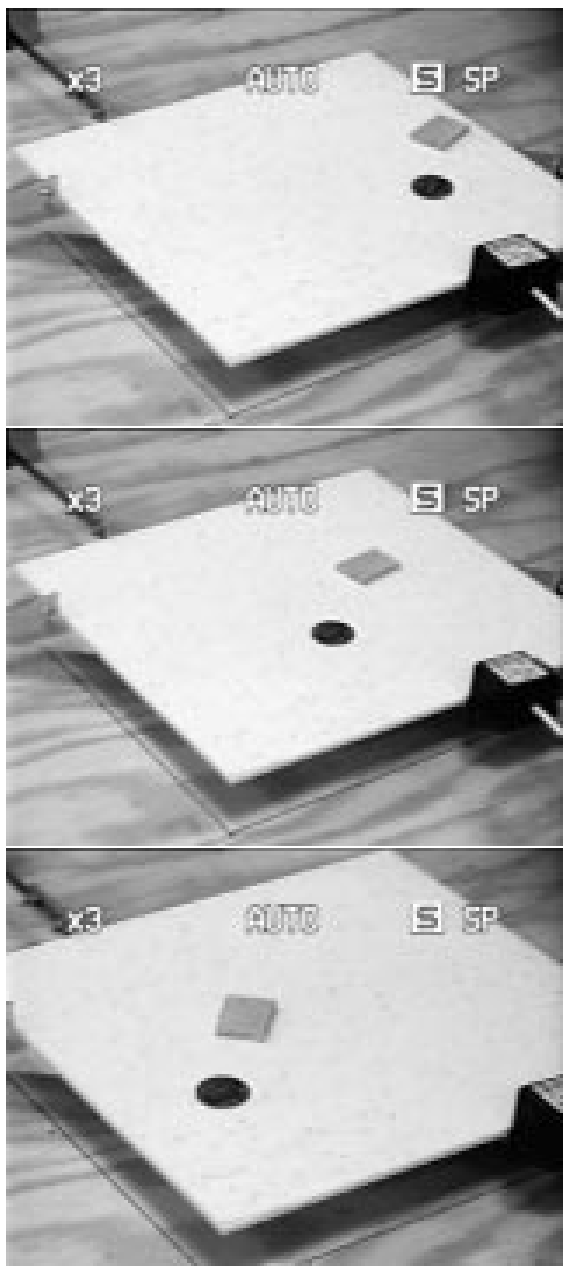

Figure 10: Three snapshots showing a penny and thin wood slab feeding along our feeder's prototype. The bang-bang acceleration method was used.

[8] N. Tran, K. Chan, and V. Hayward. Object behavior using a vibrating plate testbed for part presentation research. EE304-494 project writeup, McGill University, December 1995.

[9] K. Böhringer, V. Bhatt, and K. Goldberg. Sensorless manipulation using transverse vibrations of a plate. In IEEE International Conference on Robotics and $\mathrm{Au}$ tomation, Nagoya, Japan, May 1995.

[10] D. Reznik and J. Canny. A flat rigid plate is a universal planar manipulator. In IEEE International Conference on Robotics and Automation, Leuven, Belgium, May 1998.

[11] J. Craig. Introduction to robotics, mechanics and control. Addison-Wesley, Reading, MA, 2nd edition, 1989.

[12] M. Mason. Mechanics of Manipulation. MIT Press, Cambridge, MA, 1997.

[13] D. Reznik, J. Canny, and K. Goldberg. Analysis of part motion on a longitudinally vibrating plate. In International Workshop On Intelligent Robots and Systems, Grenoble, France, September 1997. 